\title{
Radiographic reconstruction of lower-extremity bone fragments: a first trial
}

\author{
Steffen Schumann ${ }^{1} \cdot$ Richard Bieck $^{2}$ - Rainer Bader ${ }^{2} \cdot$ Johannes Heverhagen $^{3}$. \\ Lutz-P. Nolte ${ }^{1}$ - Guoyan Zheng ${ }^{1}$
}

Received: 21 October 2015 / Accepted: 26 May 2016 / Published online: 16 June 2016

(C) CARS 2016

\begin{abstract}
Purpose The correct rotational alignment of the proximal and the distal bone fragments is an essential step in a longbone deformity correction process. In order to plan the deformity correction, plain radiographs are conventionally used. But as three-dimensional information of the complex situation is not available, the correct amount of rotation can only be approximated. Thus, the objective of this study was to develop a system to assess the rotational relationship between the proximal and distal fragments of a long bone (tibia or femur) based on a set of two calibrated X-ray radiographs. Methods In order to robustly determine the rotational relationship of proximal and distal bone fragments, a statistical shape model-based 2D/3D reconstruction approach was employed. The resulting fragment models were used to determine the angle between its anatomical axes and the rotation around its particular axes. Two different studies were performed to evaluate the accuracy of the proposed system.

Results The accuracy of the complete system was evaluated in terms of major bone axis and in-plane rotational difference. The angle between the anatomical fragment axes could be measured with an average error of $0.33^{\circ} \pm 0.27^{\circ}$, while an average in-plane rotational error of $2.27^{\circ} \pm 1.76^{\circ}$ and $2.67^{\circ}$
\end{abstract}

Steffen Schumann

steffen.schumann@ieee.org

Guoyan Zheng

guoyan.zheng@ieee.org

1 Institute for Surgical Technology and Biomechanics (ISTB), University of Bern, Bern, Switzerland

2 Biomechanics and Implant Technology Research Laboratory, Department of Orthopaedics, University of Rostock, Rostock, Germany

3 Department of Radiology, Inselspital, University of Bern, Bern, Switzerland $\pm 1.80^{\circ}$ was found for the proximal and distal fragments, respectively. The overall mean surface reconstruction error was $0.81 \pm 0.59 \mathrm{~mm}$ when the present technique was applied to the tibia and $1.12 \pm 0.87 \mathrm{~mm}$ when it was applied to the femur.

Conclusions A new approach for estimating the rotational parameters of long-bone fragments has been proposed. This approach is based on two conventional radiographs and $2 \mathrm{D} / 3 \mathrm{D}$ reconstruction technology. It is generally applicable to the alignment of any long-bone fragments and could provide an important means for achieving accurate rotational alignment.

Keywords Long bone - Tibia - Femur - Reconstruction . Radiographic $\cdot$ Distraction $\cdot$ Deformity correction $\cdot$ Fracture

\section{List of symbols}

$\begin{array}{ll}\begin{array}{l}\text { bone } \\ \text { part }\end{array} & \begin{array}{l}\text { Femur, tibia } \\ \text { bone } M_{\mathrm{gt}}\end{array} \\ \begin{array}{l}\text { Proximal, distal } \\ \text { Ground truth segmented surface model } \\ \text { bone } M_{\mathrm{gt}}\end{array} & \begin{array}{l}\text { Ground truth segmented surface model } \\ \text { (specific bone and part) }\end{array} \\ { }_{\text {part }} M_{\mathrm{rec}} & \begin{array}{l}\text { Reconstructed surface model (specific bone } \\ \text { and part) }\end{array} \\ P & \text { Set of landmarks/feature points } \\ P_{\mathrm{gt}} & \text { Set of landmarks in ground truth CT space } \\ P_{\mathrm{ssm}} & \text { Set of landmarks in statistical model space } \\ P_{\mathrm{xray}} & \text { Set of landmarks in image space } \\ P_{\text {meas }} & \text { Adjusted set of reconstructed landmarks } \\ \text { part } P n_{\mathrm{ssm}} & \text { Landmark }(n) \text { of specific part in SSM space } \\ & (n=1,2,3)\end{array}$




$$
\begin{aligned}
& \text { part } P n_{\text {rec }} \\
& \text { part } T_{\text {rec }}^{\mathrm{ssm}} \\
& \text { part } F_{\mathrm{gt}} \\
& \text { part } F_{\text {rec }} \\
& \text { part } T_{\text {rec }}^{\mathrm{ssm}} \\
& \text { dist } T_{\text {frac }}^{\text {intact }} \\
& \text { part } T_{\text {rec }}^{\mathrm{gt}} \\
& \text { part } V_{\mathrm{gt}} \\
& \text { bone } \\
& \text { part } V_{\text {rec }} \\
& \text { bone }
\end{aligned}
$$

Landmark $(n)$ of specific part in reconstructed X-ray space ( $n=1,2,3)$

Transformation of specific part from SSM- to reconstructed X-ray space

Nail fiducials identified in ground truth CT space for specific part

Triangulated nail fiducials in X-ray space for specific part

Transformation of specific fragment from SSM to reconstructed X-ray space

Transformation of distal fragment from intact to fractured status in CT space

Transformation of fragment from ground truth CT space to reconstructed X-ray space Binary volume of ground truth surface model (specific bone and part)

Binary volume of reconstructed surface model (specific bone and part)

\section{Introduction}

The correct alignment of long-bone fragments is important for the improvement of the patient's quality of life. In such a treatment, the restoration of the anatomical alignment between the proximal and the distal bone segments is an essential step. A malalignment of the bone segments can lead to an insufficient correction and a loss of limb function [1]. The alignment of bone fragments is a key step in the correction of bone deformities and in the fracture reduction process. The treatment of long-bone deformities and long-bone fractures is commonly performed with distraction osteogenesis using external fixators [2-4]. A nowadays widely used concept for distraction osteogenesis was developed by Ilizarov [5]. He invented a circular external fixator to constantly apply tension stress to the bone to stimulate its regeneration. An advancement of this system, the Taylor Spatial Frame (Smith \& Nephew, Memphis, USA), enables multidirectional deformity correction [6-8]. One of the prerequisites for a successful treatment using an external fixator is the correction of the malaligned bone segments [1]. During such a distraction phase, the patients need to undergo recurrent X-ray image acquisitions [9].

As only two-dimensional (2D) image information is available, the actual limb situation cannot be accurately assessed $[10,11]$. In order to assess the limb alignment in all three dimensions (3D), a suitable imaging modality such as computed tomography (CT) or magnetic resonance imaging (MRI) would need to be used. However, an application of these 3D imaging modalities could not be justified, as the recurrent examinations are associated with a repetitive exposure of radiation and high costs. Thus, 2D/3D reconstruction methods based on statistical shape models (SSMs) could be a valuable alternative. Methods for 2D/3D reconstruction of the femur and tibia from $\mathrm{X}$-ray radiographs were presented by Messmer et al. [12], Gunay et al. [13], Gamage et al. [14] and Quijano et al. [15]. All these approaches perform a 3D reconstruction of the intact bone and are therefore not directly applicable to the reconstruction of bone segments. In clinical practice, the X-ray radiography gives only two-dimensional information of the fractured bone. Consequently, the actual three-dimensional relationship between the proximal and distal bone fragments is not known.

The contribution of this work is twofold: (1) In order to assess the rotation of the bone fragments, $2 \mathrm{D} / 3 \mathrm{D}$ registration technique is employed to reconstruct surface models of the proximal and the distal fragments from a set of conventional $\mathrm{X}$-ray radiographs. We will present the adapted 2D/3D reconstruction framework [16] for reconstructing patient-specific surface models of both the proximal and the distal fragments of a long bone (femur or tibia) from biplanar radiographs and the determination of the rotational relationship. The advantage of using 2D/3D reconstruction technique to measure the rotation between two bone fragments over estimating it from picked landmarks lies in the 2D nature of X-ray images. As only projection information is available, precise identification of landmarks is very challenging. Thus, we propose to match $3 \mathrm{D}$ statistical shape models to the X-ray data and to derive the required landmarks from the reconstructed 3D models; (2) two studies were performed to validate the accuracy of the overall system. Digitally reconstructed radiographs (DRRs) were used in the first study to compute the reconstruction accuracy, while a customized mock-up and conventional radiography were used in the second study to evaluate the accuracy of our proposed method.

As our approach could be used for both deformity correction and fracture reduction, we will consistently use the term 'detachment site' to describe the spot of bone separation in the remainder of the manuscript. Thereby, the term 'detachment site' implies that the bone fragments could have been separated either by osteotomy during deformity correction or by traumatic fracture.

\section{Materials and methods}

\section{Statistical femur and tibia model}

For $3 \mathrm{D}$ reconstruction of a patient-specific model of a long-bone (tibia or femur) fragment, the concept of statistical shape models (SSMs) [17] is employed. Computed tomography (CT) scans of intact tibial and femoral bones were semiautomatically segmented using Amira software (FEI, Hillsboro, USA), and correspondences between the segmented surface models were determined by non-rigid mesh registration [18]. Thereby, a reference instance was 
selected and the remaining floating instances were nonrigidly registered using diffeomorphic demons algorithm [19]. For each resulting deformation field, the reference instance was accordingly warped to recover the shape of the floating instances. By this step, the correspondences among all segmented surface models were established and the shape variation could be extracted using principal component analysis (PCA). The statistical population consisted of 17 tibial and 129 femoral instances. To this end, two statistical shape models, one constructed from the 17 tibial surface models and the other constructed from 129 femoral surface models, were constructed. The statistical shape models were further used to predefine a set of landmarks on the basis of the femoral and tibial mean model (see Table 1).

\section{Definition of fragment rotation}

By using 2D/3D reconstruction technique, we are interested in describing the rotation between both bone fragments. The description of the rotation is split into two parameters: The first parameter describes the 3D angle between the anatomical axes of both fragments in its fractured status and is denoted as anatomical axis angle (AAA). The second parameter is denoted as in-plane rotation angle (IRA). It is the 2D angle describing the rotation between the proximal and the distal fragment around the anatomical axis after AAA has been compensated.
In order to quantitatively describe the rotational relationship between a pair of fragments, unique coordinate systems need to be defined for each fragment. As the shape of the fragments is not known a priori, these coordinate systems were predefined on the basis of the landmarks and feature points of the intact mean bone models. The origins of the coordinate systems (COSs) are defined by the particular (1) landmarks (see Table 1; Fig. 1). The $z$-axes are defined by connecting both proximal and distal (1) landmarks, whereas this common line also represents the main anatomical longbone axis. Thereby, the $z$-axis of the proximal COS points caudally, while the $z$-axis of the distal COS points cranially. The proximal femur $x$-axis is defined by the line passing through the origin and the femoral head center landmark as projected onto a plane, whereas its normal is corresponding to the $z$-axis. The remaining $x$-axes are defined by projecting the line out of points (2) and (3) onto a plane defined by landmark (1) (plane position) and the main long-bone axis (plane normal). For each COS, the $x$-axis points medially. The $y$ axis is derived by the cross-product of defined $z$ - and $x$-axis. The proximal $y$-axes point in anterior-posterior direction, while the distal $y$-axes are in posterior-anterior direction.

\section{D/3D reconstruction}

The 2D/3D reconstruction of the bone fragments is based on a previously developed methodology [16]. This methodol-

Table 1 Anatomical landmarks and feature points as defined on the femoral and tibial epiphysis

\begin{tabular}{cll}
\hline & Tibia & Femur \\
\hline $\begin{array}{cl}\text { Proximal } \\
(1)\end{array}$ & Center of medial and lateral intercondylar tubercle & Apex of greater trochanter \\
$(2)$ & Most medial point of the tibial plateau & Femoral head center \\
$(3)$ & Most lateral point of the tibial plateau & Apex of lower trochanter \\
Distal & & \\
$(1)$ & Center of medial malleolus and incisura fibularis & Intercondylar notch \\
$(2)$ & Apex medial malleolus & Medial epicondyle \\
$(3)$ & Center of incisura fibularis & Lateral epicondyle \\
\hline
\end{tabular}

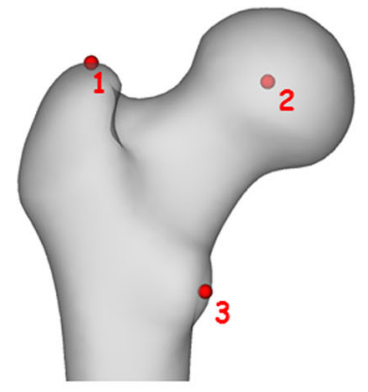

a

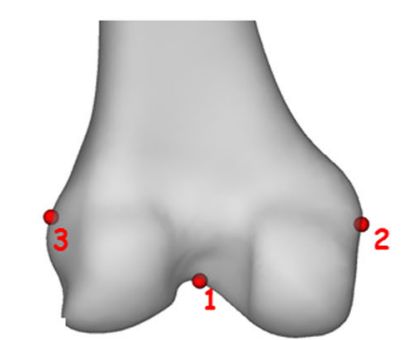

b

Fig. 1 a, b Landmarks and feature points of proximal and distal femur (left femur). The femoral head center is determined by fitting a sphere to the vertices of the surface model; $\mathbf{c}, \mathbf{d}$ tibial landmarks of proximal

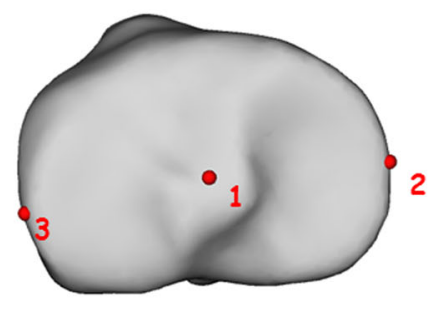

C

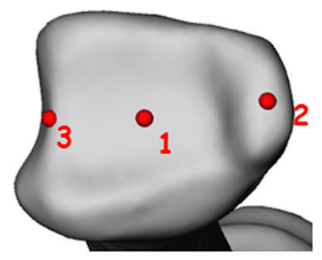

d and distal plafond (left tibia). The lateral and medial landmarks of both tibial parts are defined based on the point of maximum curvature 
ogy was originally developed for the reconstruction of the proximal femur, but can be applied for the reconstruction of any other bone. It requires a set of minimum two calibrated $\mathrm{X}$-ray images and a SSM of the particular bone to be reconstructed. Based on a set of corresponding feature points and 2D bone contours, the SSM is iteratively fit to these extracted features. Upon convergence, the SSM is instantiated based on its intrinsic statistical information. A final non-rigid deformation step is applied to optimally reconstruct the underlying bone geometry. For more details of this algorithm, we would like to refer to our previous work [16].

As the methodology of 2D/3D reconstruction follows general principles, it can be also adapted to reconstruct longbone fragments. The set of X-ray images is composed of an anterior-posterior (AP) and a lateral view of the particular bone. The calibration is accomplished by integrating a small-sized calibration unit into the acquisition process [20]. In order to initially match the SSM of the respective bone to the radiographs, the mean model needs to be split up into two independent fragments according to the present detachment site. The proximal and distal fragment lengths are estimated with respect to the length of the complete intact bone, by indicating a value between 0.0 and 1.0. A value of 0.5 indicates an approximately equal length of both fragments (detachment site at the center of the major bone axis), while values larger (smaller) than 0.5 indicate a larger proportion of the proximal (distal) fragment. Based on this value, the intact bone SSM is split into two fragments. As the SSM consists of a population of aligned surface models, each model can be split into two parts according to the indicated percentage value. It is not important to exactly match the length of the bone fragments, as we are mainly interested in the rotational arrangement of the fragments and not in the precise fragment length or the appearance of the detachment site. Both bone fragments are then independently matched to the radiographic scene based on the methodology introduced in [16].

\section{Landmark-based registration}

In the first step, corresponding landmarks and feature points $P$ need to be found, respectively, in image- and SSM spaces $\left(P_{\text {xray }}\right.$ and $\left.P_{\mathrm{ssm}}\right)$. For each bone fragment, the major bone axis and three anatomical landmarks need to be defined. In the image space, the bone axis is defined by picking two points on a virtual line along the major bone axis, whereas the exact location is not of importance as they are solely used to determine the axis. The remaining three points correspond to specific anatomical landmarks and feature points. These landmarks are commonly used in the literature [21-23] and are summarized in Table 1 and illustrated in Fig. 1.

These five points can be easily picked on the AP image. But the identification of these points is more challenging in the lateral view. Therefore, epipolar lines are visualized on the lateral image for each point identified in the AP image. Consequently, the identification of a specific point could be constrained to the particular epipolar line.

As the same features were also predefined on the intact mean model in SSM space $P_{\text {ssm }}$, unique coordinate systems could be derived for each feature set. The same coordinate systems are also used to assess the rotational relationship and are described in the next paragraph. The matching between the SSM space and the image space was achieved by equally aligning the respective pair of coordinate systems.

\section{Iterative rigid registration}

In a next step, 2D image contours were outlined using a semiautomatic method. The contours were drawn along the bone edges, whereas the detachment site was omitted. The contours have to be drawn for both bone fragments on each radiograph. Once all the contours were defined, an iterative rigid registration between the SSM and the contour data was computed to improve the alignment of the bone models to the X-ray scene.

\section{Non-rigid registration}

The remaining steps were performed as described in [16]: The aligned SSMs were instantiated and then non-rigidly deformed to recover the shape of the underlying bone fragments.

\section{Assessment of fragment rotation}

The main anatomical axis is predefined in the SSM space using two landmarks part $P 1_{\text {ssm }}$ and part $P 2_{\text {ssm }}$. In the $2 \mathrm{D} / 3 \mathrm{D}$ reconstruction pipeline, the intact mean model is loaded and subsequently split into two fragments. Both fragments are individually registered to the X-ray scene by finding the optimal transformation part $T_{\mathrm{rec}}^{\mathrm{ssm}}$. Applying both particular transformations (for each fragment) to the pair of landmarks will result in the $z$-axes of both fragments (prox $P 1_{\text {rec }}$, ${ }_{\text {dist }} P 1_{\text {rec }}$, prox $P 2_{\text {rec, dist }} P 2_{\text {rec }}$ ). In the same way, the remaining landmarks are retrieved for both reconstructed fragments. The $x$ - and $y$-axes of the coordinate systems are then computed based on the anatomical axis as retrieved for each fragment (see section "Materials and methods"). The angle between the $z$-axes of both particular bone fragments describes the anatomical axis angle (AAA), which would need to be compensated for in order to restore the original long-bone anatomical axis. After restoring the long-bone anatomical axis, the rotation around this axis between the proximal and distal fragment is described by the $x$ - and $y$ axes. 


\section{DRR-study}

1. Segmentation of ground truth CTdatasets

2. Identification of ground truth landmarks

3. Split up CT-volume into two parts

4. Generation of fractured CT-volumes:

Transformation of distal fragment

dist $T_{\text {frac }}^{\text {intact }}$

5. Generation of biplanar DRRs from

fractured volume

6. $2 D / 3 D$ reconstruction of proximal and distal fragments

\section{Mockup-study}

1. Segmentation of ground truth CT-dataset in intact fragment configuration

2. Identification of ground truth landmarks and fiducials

3. Radiography of mockup with fragments in particular fracture configuration

4. $2 \mathrm{D} / 3 \mathrm{D}$ reconstruction of proximal and distal fragments and multiview reconstruction of projected fiducials

5. Alignment of ground truth fragments to reconstructed fragments based on fiducials; determination of distal fragment transformation from intact to fractured state dist $_{\text {frac }}^{\text {intact }}$

6. Rigid alignment of ground truth fragments to reconstructed fragments ${ }_{\text {frag }} T_{\text {rec }}^{g T}$

7. Crop fragments at same level and compute surface reconstruction error

8. Generate binary volume for each fragment model

9. Non-rigid registration of ground truth and reconstructed volumes $=>$ displacement fields

10. Identification of reconstructed landmarks using displacement fields

11. Align reconstructed distal fragment to proximal fragment based on inverse ${ }_{\text {dist }} T_{\text {frac }}^{\text {intact }}$

12. Set up specific coordinate-systems based on landmarks for both models

13. Compute alignment errors

Fig. 2 Series of steps carried out to analyze the accuracy of the proposed system for both studies. Steps (7) to (13) were carried out for both studies

\section{Experimental validation}

As part of this experimental validation, two studies were conducted to evaluate the accuracy of our proposed method. In a first study, DRRs of virtually detached femur and tibia bones were used to validate the proposed approach. In a second study, a mock-up phantom was constructed to simulate different malalignments. Conventional radiographs of different configurations were acquired, and the fragments were reconstructed as described in section "2D/3D reconstruction". The landmarks, contours and the detachment site were identified by the respective operator. For both studies, the 2D/3D reconstruction accuracy in terms of surface error and the rotational errors was assessed. The rotational errors were determined by comparing the reconstructed values for AAA and IRA with the ground truth data. A summary of all involved steps is shown in Fig. 2.

\section{Experimental validation using DRRs}

In this first study, CT datasets of cadaveric specimens (pixel dimensions: $512 \times 512 \times 875$; voxel size: $1.27 \mathrm{~mm} \times$ $1.27 \mathrm{~mm} \times 0.5 \mathrm{~mm}$ ) were segmented using Amira software and surface models of the femur and tibia bone $M_{\mathrm{gt}}$; bone $=$ femur, tibia were extracted (step 1 of DRR study in Fig. 2). For each segmented surface model, ground truth landmarks and feature points $P_{\mathrm{gt}}$ as defined in Table 1 (see also
Fig. 1) were manually defined (step 2 in Fig. 2). Moreover, three different detachment levels were simulated for each bone by splitting the CT volume into a proximal and a distal half ( ${ }_{\text {bone }}^{\text {part }} M_{\mathrm{gt}}$; part = prox, dist) (step 3 in Fig. 2). These three levels were approximately in both metaphysis regions (proximal and distal) and at the center of the diaphysis. In order to test different limb deformity scenarios, randomly composed transformations were applied to the distal half of the split CT volume $\underset{\text { bone }}{\text { dist }} M_{\mathrm{gt}}$ (step 4 in Fig. 2). The translation along the transversal axis was in the range of $20 \mathrm{~mm}$ (cranially) and $50 \mathrm{~mm}$ (caudally). The translation of the fragment in the transverse plane was in the range of $\pm 75 \mathrm{~mm}$. The rotation around the transverse axis was in the range of $\pm 75^{\circ}$ and $\pm 45^{\circ}$ around the sagittal and coronal axis. The resulting composed CT volume was further used to generate two DRRs in AP and lateral view (step 5 in Fig. 2). The DRRs were generated with a film focal distance of $1600 \mathrm{~mm}$ (film object distance of $100 \mathrm{~mm}$ ), an image size of $2048 \times 2048$ pixels and a pixel spacing of $0.25 \mathrm{~mm}$. Subsequently, the DRRs with corresponding calibration information were used for 2D/3D reconstruction as described in section " $2 \mathrm{D} / 3 \mathrm{D}$ reconstruction" (step 6 in Fig. 2). The reconstructed models $\underset{\text { bone }}{\mathrm{part}} M_{\text {rec }}$ were consequently in the same space as the ground truth models. A set of DRRs and reconstructed models is shown in Fig. 3. The analysis of these data is further explained in section "Subjective-free validation", whereas a summary of all involved steps is shown in Fig. 2. 


\section{Experimental validation using mock-up phantom}

In this second study, conventional radiographs of a customized mock-up were acquired. This mock-up was designed and constructed for the purpose of simulating various malalignments. The malalignments were manually adjusted and recorded with a tracking camera (Polaris, NDI, Ontario, Canada). Therefore, both fragments were equipped with dynamic reference bases. The mock-up further provides a separate fixation for two plastic bone fragments and allows to freely adjust both fragments (see Fig. 4). While the proximal fragment is rigidly attached to a fixation block which can be varied in its height and distance on the base plate, the distal fragment is connected to the base plate via a modular hose system (Lockwood Products Inc., Lake Oswego, USA). The hose system enables a nearly unrestricted spatial positioning of the distal bone fragment with respect to the proximal bone fragment as well as $360^{\circ}$ of rotation around the main anatomical axis. Moreover, the calibration unit was rigidly attached to the base plate. All the mock-up components were made out of plastic material (PMMA) to preserve radiolucent properties of the whole setup. Six nail fiducials with tiny indents were inserted into each bone fragment for accuracy analysis. The exact fiducial locations are summarized in Table 2 and illustrated in Fig. 5.

The mock-up was consecutively equipped with fragments of two different tibial plastic bones. Both plastic bones were artificially detached by sawing a wedge-shaped profile into the diaphysis. For the first bone, the detachment site was laterally oriented and more proximally (see Fig. 4), while the detachment site of the second bone was more distally and in anterior-posterior direction. After mounting each bone to the mock-up, the fragments were realigned and fixed, recovering the original intact tibia bone for acquisition of a ground truth CT scan (Somatom, Siemens, Erlangen, Germany). The CT datasets of both bones were semiautomatically segmented using Amira software, resulting in ground truth model fragments bone $M_{\mathrm{gt}}$ (step 1 of mock-up study in Fig. 2). Moreover, the nail fiducials part $F_{\mathrm{gt}}$ (see Table 2; Fig. 5) were identified by manually localizing the fiducial center in the respective CT stack (step 2 in Fig. 2). Thereupon, the fragments were manually put into five different configurations, simulating different tibial malalignments. For each configuration, two $\mathrm{X}$-ray radiographs (AP and lateral view) were acquired and consequently calibrated (step 3 in Fig. 2). The set of calibrated radiographs was used to reconstruct the proximal and distal fragments $\underset{\text { bone }}{\text { part }} M_{\text {rec }}$ (step 4 in Fig. 2). In addition, the projected nail fiducials were identified in both views and triangulated to unique 3D positions part $F_{\text {rec }}$. Pair-point matching between part $F_{\text {gt }}$ and part $F_{\text {rec }}$ was performed to align the ground truth fragments with the particular configuration in X-ray space (step 5 in Fig. 2). Thus, corresponding nail fiducials were used to compute the respective transformation. In order to analyze the surface reconstruction accuracy, a rigid registration for each corresponding pair of ground

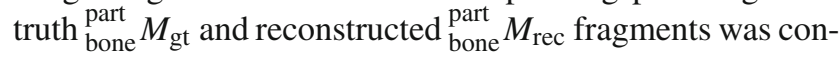
ducted (step 6 in Fig. 2). Upon registration, the data were further analyzed as described in section "Subjective-free validation".
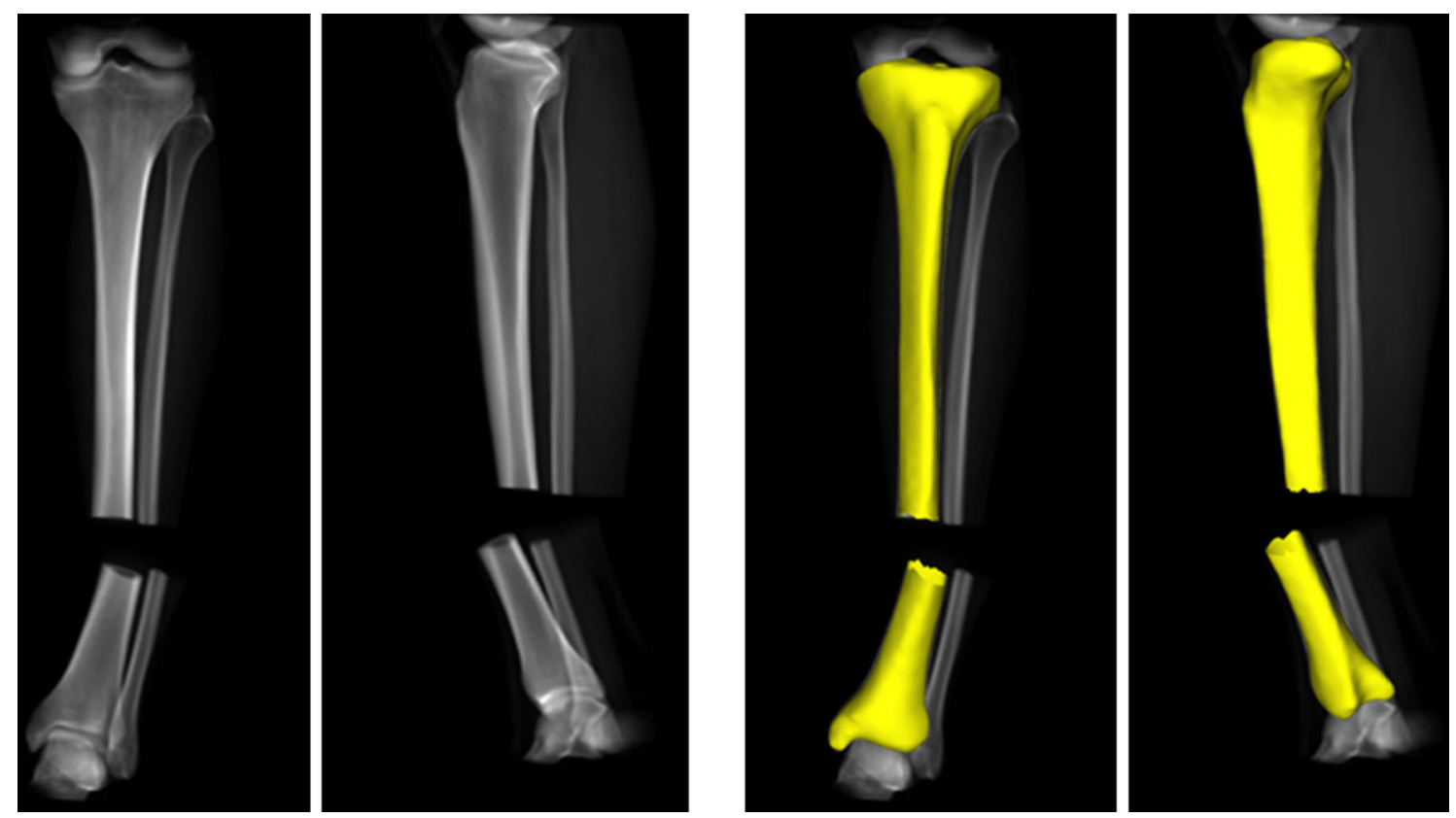

Fig. 3 Set of digitally reconstructed radiographs (left) and with reconstructed fragments superimposed (right) 


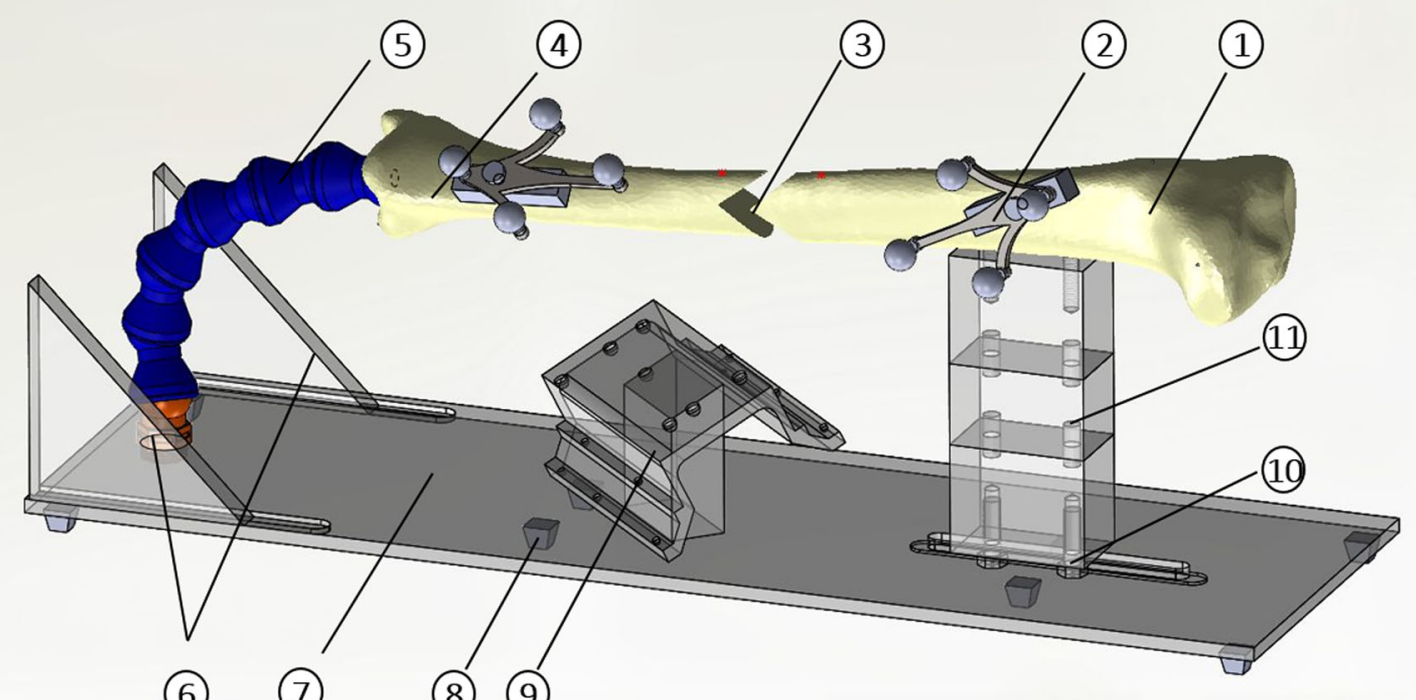

(6) (7) (8) (9)

Fig. 4 Left drawing of the constructed mock-up: 1 proximal bone fragment, 2 proximal dynamic reference base, 3 detachment site, 4 distal bone fragment, 5 modular positioning arm, 6 vertical positioning sup- port, 7 base plate, 8 anti-slip posts, 9 calibration unit, 10 horizontal adjustment, 11 vertical adjustment
Table 2 Anatomical location of fiducials inserted into both fragments of each tibia bone model

\begin{tabular}{ll}
\hline Proximal & Distal \\
\hline Proximal fragment & \\
1. Tuberositas tibia & 4. Margo interosseous \\
2. Lateral tibia plateau edge & 5. Margo anterior \\
3. Medical tibia plateau edge & 6. Margo medialis \\
Distal fragment & \\
7. Margo interosseous & 10. Apex malleolus medialis \\
8. Margo anterior & 11. Anterior end of incisura fibularis \\
9. Margo medialis & 12. Posterior end of incisura fibularis
\end{tabular}
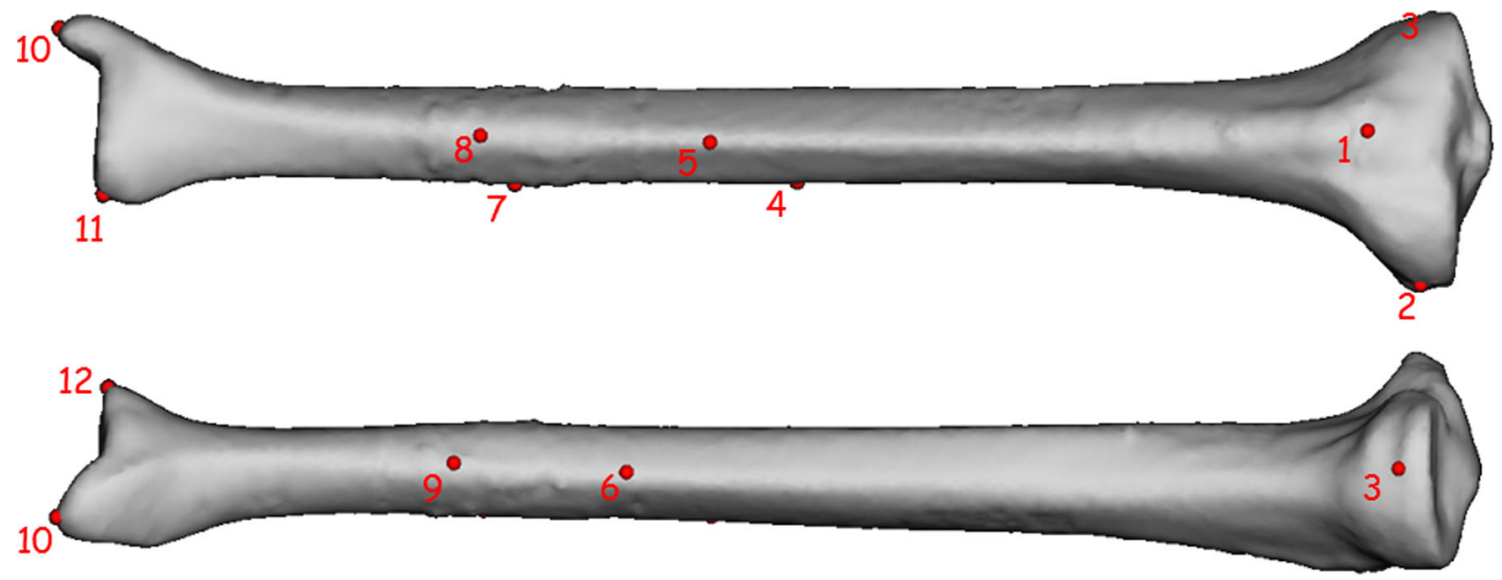

Fig. 5 Specific locations of nail fiducials used for matching the CT and radiographic scene 


\section{Subjective-free validation}

Each ground truth fragment is now in the same space as its reconstructed counterpart. As the length of the reconstructed fragments was not exactly equal to the actual ground truth length, the pair of corresponding models were cropped at the same level near the detachment site. The surface reconstruction error was then computed between the pair of equally cropped fragments (step 7 in Fig. 2). The rotational error was assessed based on the ground truth $P_{\mathrm{gt}}$ and reconstructed landmarks and feature points $P_{\text {rec. }}$. As the reconstructed model ${ }_{\text {bone }}^{\text {part }} M_{\text {rec }}$ is subject to certain small distortions during the non-rigid registration process, landmark information $P_{\text {rec }}$ could not be taken over from the original statistical mean model and thus would need to be redefined. One option would be to manually redefine the landmark positions on the reconstructed bone models. Thus, in clinical practice, this step would need to be done by the surgeon. But for the sake of validating the assessment of the fragment rotation, the landmark positions were instead inferred from the ground truth models (which though would not be available in clinical practice) using a non-rigid registration step. By this step, the subjective landmark picking process could be omitted: Firstly, both cropped surface models $\underset{\text { bone }}{\text { part }} M_{\mathrm{gt}}$ and ${ }_{\text {bone }}^{\text {part }} M_{\text {rec }}$ were transformed to binary volumes ${ }_{\text {bone }}^{\text {part }} V_{\mathrm{gt}}$ and ${ }_{\text {bone }}^{\text {part }} V_{\text {rec }}$ using Amira software (step 8 in Fig. 2). Subsequently, diffeomor- phic demons algorithm [19] was used to non-rigidly deform the ground truth volume $\underset{\text { bone }}{\text { part }} V_{\mathrm{gt}}$ with respect to the reconstructed volume $\underset{\text { bone }}{\text { part }} V_{\text {rec }}$ (step 9 in Fig. 2). The computed displacement vector field was then applied to the ground truth feature points $P_{\mathrm{gt}}$, which had been updated by the rigid transformation frag $T_{\text {rec }}^{\mathrm{gt}}$. The resulting feature points were further adjusted to correspond to the closest vertex on the reconstructed model, resulting in the actually reconstructed feature points $P_{\text {meas }}$ (step 10 in Fig. 2). In order to determine the reconstruction accuracy of the main long-bone axis, the pair of reconstructed fragments ${ }_{\text {bone }}^{\text {prox }} M_{\mathrm{gt}}$ and ${ }_{\text {bone }}^{\text {dist }} M_{\mathrm{gt}}$ were aligned to recover the intact bone configuration (step 11 in Fig. 2). Thereby, the ground truth transformation of the distal fragment from intact to detached state was inverted and applied to the reconstructed distal fragment.

The reconstructed landmarks and feature points $P_{\text {meas }}$ were then used to set up the coordinate systems for each fragment, as described in "Assessment of fragment rotation" (step 12 in Fig. 2). These reconstructed COSs were then compared to the ground truth COSs in terms of two error measurements (step 13 in Fig. 2). The anatomical axis error (AAE) is measured as the difference between the anatomical axis angle (AAA) of the reconstructed and the ground truth situation. The in-plane rotation error (IRE) is computed as the in-plane rotation angle (IRA) of the reconstructed and the ground truth situation.
Table 3 Surface mean and root-mean-square error in $\mathrm{mm}$ for each trial of the DRR study

\begin{tabular}{|c|c|c|c|c|c|c|c|c|}
\hline \multirow[t]{3}{*}{ Trial ID } & \multicolumn{4}{|l|}{ Tibia } & \multicolumn{4}{|c|}{ Femur } \\
\hline & \multicolumn{2}{|l|}{ Prox. } & \multicolumn{2}{|l|}{ Dist. } & \multicolumn{2}{|l|}{ Prox. } & \multicolumn{2}{|l|}{ Dist. } \\
\hline & Mean & RMS & Mean & RMS & Mean & RMS & Mean & RMS \\
\hline 1 & 0.95 & 1.14 & 1.32 & 1.67 & 0.88 & 1.11 & 1.10 & 1.41 \\
\hline 2 & 0.94 & 1.19 & 0.63 & 0.81 & 0.98 & 1.27 & 1.10 & 1.38 \\
\hline 3 & 0.93 & 1.19 & 0.62 & 0.76 & 1.07 & 1.33 & 1.12 & 1.38 \\
\hline 4 & 1.12 & 1.28 & 0.84 & 1.01 & 0.99 & 1.25 & 1.31 & 1.66 \\
\hline 5 & 1.00 & 1.20 & 0.95 & 1.14 & 0.93 & 1.17 & 1.65 & 2.01 \\
\hline 6 & 0.84 & 1.01 & 0.93 & 1.09 & 1.20 & 1.61 & 1.13 & 1.35 \\
\hline 7 & 0.86 & 1.09 & 0.93 & 1.11 & 1.14 & 1.42 & 0.94 & 1.21 \\
\hline 8 & 0.83 & 0.99 & 0.76 & 0.92 & 1.11 & 1.46 & 0.85 & 1.07 \\
\hline 9 & 0.75 & 0.94 & 0.81 & 1.01 & 0.91 & 1.16 & 0.90 & 1.12 \\
\hline 10 & 0.84 & 1.04 & 1.00 & 1.26 & 0.94 & 1.19 & 0.58 & 0.72 \\
\hline 11 & 0.90 & 1.13 & 0.81 & 0.99 & 1.28 & 1.66 & 0.71 & 0.90 \\
\hline 12 & 0.89 & 1.12 & 0.52 & 0.69 & 1.43 & 1.84 & 0.84 & 1.06 \\
\hline 13 & 0.70 & 0.89 & 0.69 & 0.85 & 1.09 & 1.40 & 1.68 & 2.09 \\
\hline 14 & 0.83 & 1.02 & 0.72 & 0.88 & 1.32 & 1.62 & 1.39 & 1.76 \\
\hline 15 & 0.99 & 1.26 & 0.55 & 0.67 & 1.49 & 1.93 & 1.22 & 1.53 \\
\hline 16 & 0.80 & 1.00 & 0.72 & 0.88 & 0.92 & 1.18 & 1.40 & 1.74 \\
\hline 17 & 0.88 & 1.05 & 0.70 & 0.87 & 1.16 & 1.52 & 1.13 & 1.34 \\
\hline 18 & 0.87 & 1.07 & 0.58 & 0.72 & 1.26 & 1.62 & 1.25 & 1.55 \\
\hline Avg. & 0.88 & 1.09 & 0.78 & 0.96 & 1.12 & 1.43 & 1.13 & 1.40 \\
\hline
\end{tabular}




\section{Results}

\section{Results of DRR study}

Six femoral and six tibial CT datasets were used in the DRR study. For each CT dataset, three differently detached volumes were generated, resulting in 36 datasets in total. The respective CT datasets were not part of the population, used to generate the statistical models. In the first step, the surface reconstruction error was computed. On average, the proximal tibia fragment was reconstructed with an error of $0.88 \pm 0.63 \mathrm{~mm}$ and the distal tibia fragment with an error of $0.78 \pm 0.56 \mathrm{~mm}$. The surface reconstruction error of the femoral fragments was slightly increased. An error of $1.12 \pm 0.89 \mathrm{~mm}$ was observed for the proximal fragment, while the distal fragment was reconstructed with an error of $1.13 \pm 0.84 \mathrm{~mm}$. The mean and root-mean-square (RMS) are shown in Table 3. The anatomical axis error (AAE) was relatively robust throughout all 36 cases. For the tibia reconstructions, an average $\mathrm{AAE}$ of $0.24^{\circ} \pm 0.17^{\circ}$ (maximum error of $0.80^{\circ}$ ) was found, while the average AAE was $0.25^{\circ} \pm 0.14^{\circ}$ (maximum error of $0.80^{\circ}$ ) for the femur reconstructions. The average in-plane rotation error (IRE) was independently assessed for the proximal and distal fragments. The IRE of the proximal tibia fragments was $3.08^{\circ} \pm 2.01^{\circ}$ (maximum error of $7.65^{\circ}$ ) and $3.16^{\circ} \pm 2.02^{\circ}$ (maximum error of $7.53^{\circ}$ ) for the distal tibia fragments. In contrast to the surface reconstruction error, the IRE was improved for the femoral fragments. $1.32^{\circ} \pm 0.95^{\circ}$ (max. error $3.22^{\circ}$ ) was measured for the proximal IRE and $2.71^{\circ} \pm 1.69^{\circ}$ (max. error of $6.61^{\circ}$ ) for the distal IRE. The individual contributions to the average error are shown in Fig. 6.

\section{Results of mock-up study}

Two different tibial bone models were used to simulate ten different malalignments (five for each model). The applied translation was in the range of approximately $\pm 40 \mathrm{~mm}$ in transverse direction and around $\pm 35 \mathrm{~mm}$ within the transverse plane. The applied rotation was in the range of $\pm 20^{\circ}$ around the transverse axis and around $\pm 30^{\circ}$ around the sagittal and coronal axis. The average surface reconstruction error of the proximal fragment was $0.71 \pm 0.59 \mathrm{~mm}$ $(0.74 \pm 0.60 \mathrm{~mm}$ for the first tibia model and $0.68 \pm 0.57 \mathrm{~mm}$ for the second tibia model). The average surface reconstruction error of the distal fragment was $0.78 \pm 0.67 \mathrm{~mm}$ and thus in a similar range as for the proximal fragment. For the first

Table 4 Surface mean and root-mean-square error in $\mathrm{mm}$ for each trial of the mock-up study

\begin{tabular}{llllll}
\hline Trial ID & Prox. & & & \multicolumn{2}{l}{ Dist. } \\
\cline { 2 - 3 } \cline { 5 - 6 } & Mean & RMS & & Mean & RMS \\
\hline 1 & 0.68 & 0.87 & & 0.75 & 0.98 \\
2 & 0.65 & 0.85 & 0.82 & 1.06 \\
3 & 0.95 & 1.22 & 0.79 & 1.05 \\
4 & 0.73 & 0.94 & 0.73 & 0.96 \\
5 & 0.68 & 0.86 & 0.84 & 1.07 \\
6 & 0.65 & 0.84 & 0.87 & 1.13 \\
7 & 0.65 & 0.87 & 0.81 & 1.10 \\
8 & 0.62 & 0.82 & 0.66 & 0.86 \\
9 & 0.72 & 0.94 & 0.71 & 0.99 \\
10 & 0.76 & 0.98 & 0.82 & 1.10 \\
Avg. & 0.71 & 0.92 & 0.78 & 1.03 \\
\hline
\end{tabular}

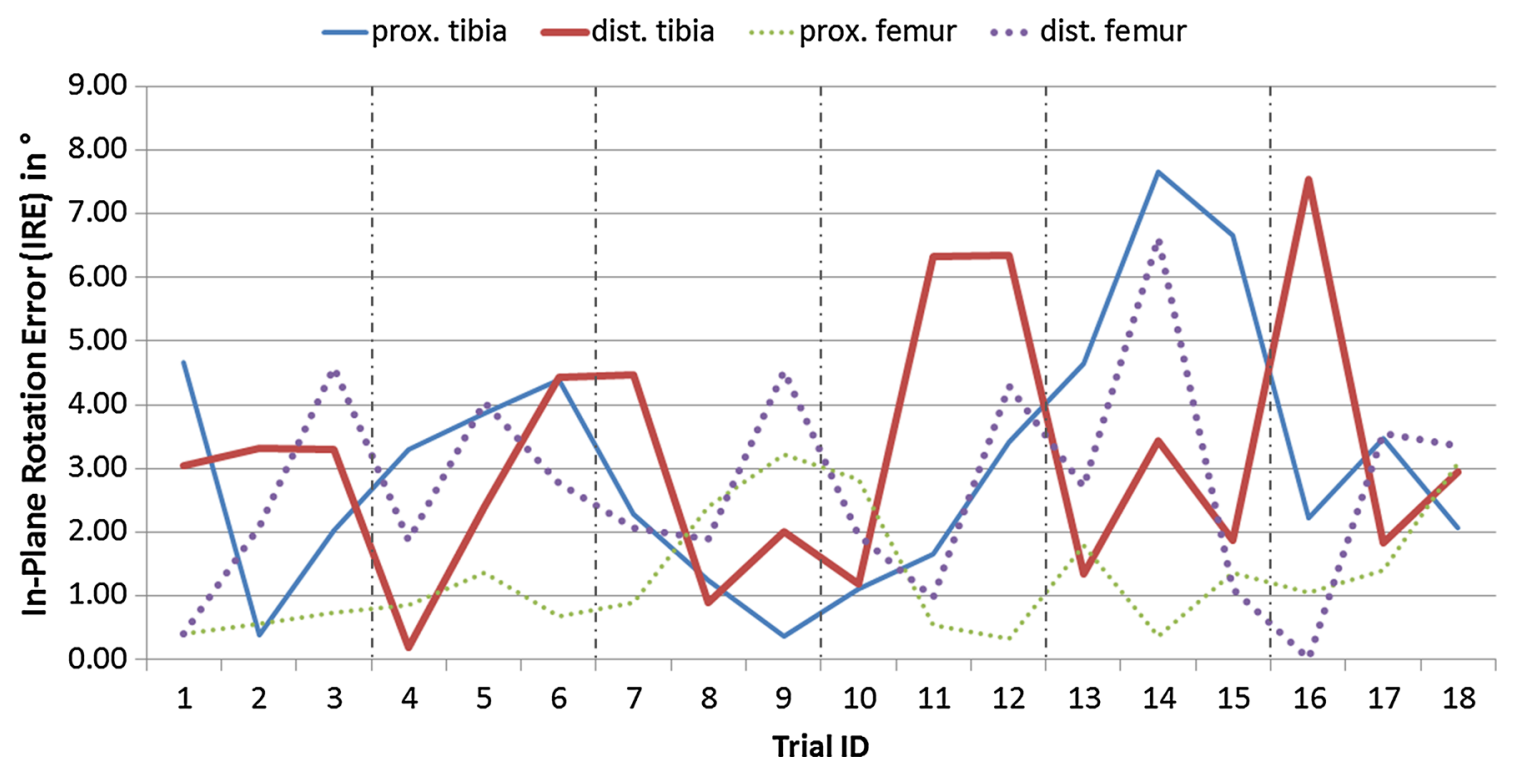

Fig. 6 In-plane rotation errors of DRR study. The femoral IREs are depicted by dotted lines. Three successive trials belong to the same bone 
Fig. 7 In-plane rotation error of the mock-up study. The first five cases belong to the first tibia model and the second five cases to the second tibia model. The proximal tibia IRE is depicted by a discontinuous line

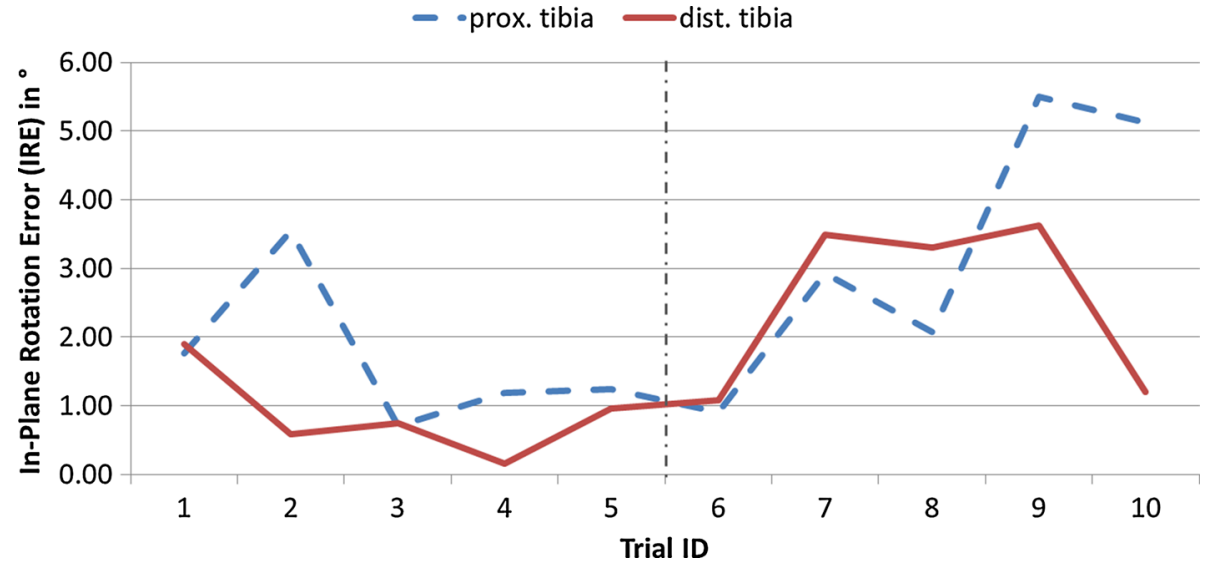

tibia model, the error was $0.79 \pm 0.66$ and $0.77 \pm 0.69 \mathrm{~mm}$ for the second bone. The individual errors are shown in Table. 4. Major inaccuracies were distributed around the tibial plateau and the plafond area. Thereby, prominent bone edges such as malleolus medialis or the eminentia intercondylaris showed the highest errors.

The AAE of the main tibial axis was on average $0.48^{\circ} \pm$ $0.38^{\circ}\left(0.41^{\circ} \pm 0.25^{\circ}\right.$ for the first tibia model and $0.36^{\circ} \pm$ $0.87^{\circ}$ for the second tibia model). The IRE observed for the proximal fragment was $2.50^{\circ} \pm 1.73^{\circ}\left(1.69^{\circ} \pm 1.10^{\circ}\right.$ for the first model and $3.31^{\circ} \pm 1.97^{\circ}$ for the second model) and $1.70^{\circ} \pm 1.30^{\circ}\left(0.87^{\circ} \pm 0.64^{\circ}\right.$ for the first tibia and $2.54^{\circ} \pm$ $1.28^{\circ}$ for the second tibia) for the distal fragment. Maximum errors of $5.50^{\circ}$ and $5.12^{\circ}$ were measured for two trials of the second proximal model, slightly exceeding the clinically acceptable error of $5^{\circ}$. The contribution of each trial to this average in-plane rotation error is shown in Fig. 7.

\section{Discussion}

The correct alignment of bone fragments is a crucial step in the surgical correction of limb deformities and in the process of fracture reduction. The alignment of bone fragments during the distraction process is monitored by the acquisition of $\mathrm{X}$-ray radiographs at regular intervals. As only 2D information is available, the assessment of the actual 3D fragment alignment is limited. For a precise planning of deformity correction and fracture reduction, it is important to understand the present scenario in 3D. In order to assess the rotation of long-bone fragments in 3D, we developed a robust method based on biplanar plain X-ray radiographs. A SSM-based 2D/3D reconstruction algorithm was adapted to reconstruct patient-specific detached femoral and tibial bones. Two studies based on DRRs and conventional X-ray radiographs were conducted to evaluate the accuracy of the proposed approach.

The mean surface reconstruction error for the femoral fragments was $1.12 \pm 0.87 \mathrm{~mm}$ (18 femoral fragment recon- structions in total) and $0.80 \pm 0.61 \mathrm{~mm}$ for the tibial fragments $(18+10$ tibial fragment reconstructions in total). Major errors were thereby found at the greater trochanter and the tibial plafond area. In comparison with other existing works on 2D/3D femur and tibia reconstruction, the presented surface reconstruction errors are considerably lower. While Messmer et al. [12] achieved average errors of $1.5 \pm 0.70 \mathrm{~mm}$ for the tibial shaft, $2.2 \pm 0.79 \mathrm{~mm}$ for the tibial plateau and $2.4 \pm 0.82 \mathrm{~mm}$ for the plafond area, Quijano et al. [15] reported a mean reconstruction error of $1.3 \mathrm{~mm}$.

Besides the surface reconstruction error, the geometrical error was assessed in terms of angular difference of anatomical axes and transversal in-plane rotation difference. With the proposed method, the long-bone axis could be derived with a $3 \mathrm{D}$ angular error of $0.33^{\circ} \pm 0.27^{\circ}$. The rotational error around this long-bone axis was on average $2.27^{\circ} \pm 1.76^{\circ}$ for the proximal bone fragments and $2.67^{\circ} \pm 1.80^{\circ}$ for the distal bone fragments. Thus, the average rotational error was clearly below the maximum clinical acceptable error of $5^{\circ}$ for both fragments. The reconstruction of the major axis was generally robust, while the rotational error around this axis was slightly higher in comparison. This observation could be explained by the $2 \mathrm{D} / 3 \mathrm{D}$ reconstruction process. While a tight fitting of the SSM to the outer contours is always achieved, certain rotations around this main longitudinal axis cannot be fully recovered from two views.

We also have to acknowledge certain limitations of the presented method. An explicit reconstruction of the detachment site and the correct bone fragment length was not considered. Moreover, the focus was on a system to assess the rotational relationship between two major bone fragments, which are detached in the diaphysis region. Consequently, the method is not applicable to comminuted fractures. In addition, the proposed method is operator-dependent as it relies on manually defined landmarks and contours. The 3D reconstruction process is particularly sensitive to the identification of the landmarks and feature points. A misplaced landmark $P_{\text {xray }}$ could lead to a certain rotational reconstruction error. This 
is due to the fact that only the proximal and distal ends of a long bone have prominent features to control the registration around its longitudinal axis. Another limitation of our study is the relatively small training population of the tibial statistical model and the low number of specimens. Thus, more data need to be gathered to draw a clinically meaningful conclusion.

The proposed concept of deriving the axial and rotational relationship of major bone fragments could be applied to any long bone. Thus, the 3D rotation between major bone fragments could always be quantitatively determined. Moreover, the proposed system of 2D/3D reconstruction and fragment alignment has the potential to reduce the number of X-ray radiographs, required to plan and monitor the distraction osteogenesis.

Acknowledgments The authors would like to thank Alper Akcöltekin, Urs Rohrer and the team from the machine shop for the valuable support in conducting this study.

\section{Compliance with ethical standards}

Conflict of interest The authors have no conflict of interest related to this work.

\section{References}

1. Kanellopoulos A, Soucacos P (2006) Management of nonunion with distraction osteogenesis. Injury 37(1):S51-S55

2. Paley D (2002) Principles of deformity correction. Springer, New York

3. Tsuchiya H, Tomita K (2003) Distraction osteogenesis for treatment of bone loss in the lower extremity. J Orthop Sci 8(1):116-124

4. GugenheimJr JJ, Brinker MR (2003) Bone realignment with use of temporary external fixation for distal femoral valgus and varus deformities. J Bone Joint Surg 85(7):1229-1237

5. Ilizarov GA (1990) Clinical application of the tension-stress effect for limb lengthening. Clin Orthop Relat Res 250:8-26

6. Feldman David S, Shin Steven S, Madan Sanjeev, Koval Kenneth J (2003) Correction of tibial malunion and nonunion with sixaxis analysis deformity correction using the taylor spatial frame. $\mathbf{J}$ Orthop Trauma 17(8):549-554

7. Fadel Mohamed, Hosny Gamal (2005) The taylor spatial frame for deformity correction in the lower limbs. Int Orthop 29(2):125-129

8. Manner Hans Michael, Huebl Michael, Radler Christof, Ganger Rudolf, Petje Gert, Grill Franz (2007) Accuracy of complex lowerlimb deformity correction with external fixation: a comparison of the taylor spatial frame with the ilizarov ring fixator. J Child Orthop 1(1):55-61
9. Paley D (2014) Progress in and from Limb Lengthening. Tree Life Media, India

10. Durandet A, Ricci P-L, Saveh AH, Vanat Q, Wang B, Esat I, Chizari M (2013) Radiographic analysis of lower limb axial alignments. In: Proceedings of the world congress on engineering, vol 2

11. Cobb JP, Dixon H, Dandachli W, Iranpour F (2008) The anatomical tibial axis reliable rotational orientation in knee replacement. $\mathrm{J}$ Bone Joint Surg Br 90(8):1032-1038

12. Messmer P, Long G, Suhm N, Regazzoni P, Jacob AL (2001) Volumetric model determination of the tibia based on $2 \mathrm{~d}$ radiographs using a 2d/3d database. Comput Aided Surg 6(4):183-194

13. Gunay M, Shim M-B, Shimada K (2007) Cost- and time-effective three-dimensional bone-shape reconstruction from X-ray images. Int J Med Robot Comput Assist Surg 3(4):323-335

14. Gamage Pavan, Xie Sheng Quan, Delmas Patrice, Xu Wei Liang (2011) Diagnostic radiograph based 3d bone reconstruction framework: application to the femur. Comput Med Imaging Graph 35(6):427-437

15. Quijano S, Serrurier A, Aubert B, Laporte S, Thoreux P, Skalli W (2013) Three-dimensional reconstruction of the lower limb from biplanar calibrated radiographs. Med Eng Phys 35(12):1703-1712

16. Zheng G, Schumann S (2009) 3d reconstruction of a patientspecific surface model of the proximal femur from calibrated X-ray radiographs: a validation study. Med Phys 36(4):1155-1166

17. Cootes TF, Taylor CJ, Cooper DH, Graham J (1995) Active shape models-their training and application. Comput Vis Image Underst 61(1):38-59

18. Heitz G, Rohlfing T, Maurer CR Jr (2005) Statistical shape model generation using nonrigid deformation of a template mesh. Med Imaging, International Society for Optics and Photonics, pp 14111421

19. Vercauteren T, Pennec X, Perchant A, Ayache N (2009) Diffeomorphic demons: efficient non-parametric image registration. NeuroImage 45(1):S61-S72

20. Schumann S, Thelen B, Ballestra S, Nolte LP, Büchler P, Zheng G (2014) X-ray image calibration and its application to clinical orthopedics. Med Eng Phys 36(7):968-974

21. Victor J, Van Doninck D, Labey L, Innocenti B, Parizel PM, Bellemans J (2009) How precise can bony landmarks be determined on a ct scan of the knee? Knee 16(5):358-365

22. Subburaj K, Ravi B, Agarwal M (2009) Automated identification of anatomical landmarks on $3 \mathrm{~d}$ bone models reconstructed from ct scan images. Comput Med Imaging Graph 33(5):359-368

23. Wu G, Siegler S, Allard P, Kirtley C, Leardini A, Rosenbaum D, Whittle M, DLima DD, Cristofolini L, Witte H, Schmid O, Stokes I (2002) Isb recommendation on definitions of joint coordinate system of various joints for the reporting of human joint motionpart i: ankle, hip, and spine. J Biomech 35(4):543-548 